\title{
Bogoliubov theory of quantum correlations in the time-dependent Bose-Hubbard model
}

\author{
Uwe R. Fischer, ${ }^{1}$ Ralf Schützhold, ${ }^{2}$ and Michael Uhlmann ${ }^{2,3}$ \\ ${ }^{1}$ Eberhard-Karls-Universität Tübingen, Institut für Theoretische Physik, Auf der Morgenstelle 14, D-72076 Tübingen, Germany \\ ${ }^{2}$ Institut für Theoretische Physik, Technische Universität Dresden, D-01062 Dresden, Germany \\ ${ }^{3}$ Department of Physics, Australian National University, Canberra, ACT 0200, Australia
}

(Received 7 December 2007; revised manuscript received 6 March 2008; published 14 April 2008)

\begin{abstract}
By means of an adapted mean-field expansion for large fillings $n \gg 1$, we study the evolution of quantum fluctuations in the time-dependent Bose-Hubbard model, starting in the superfluid state and approaching the Mott phase by decreasing the tunneling rate or increasing the interaction strength in time. For experimentally relevant cases, we derive analytical results for the temporal behavior of the number and phase fluctuations, respectively. This allows us to calculate the growth of the quantum depletion and the decay of off-diagonal long-range order. We estimate the conditions for the observability of the time dependence in the correlation functions in the experimental setups with external trapping present. Finally, we discuss the analogy to quantum effects in the early universe during the inflationary epoch.
\end{abstract}

DOI: 10.1103/PhysRevA.77.043615 PACS number(s): 03.75.Lm, 73.43.Nq, 03.75.Kk, 05.70.Fh

\section{INTRODUCTION}

The rapidly growing interest in the exploration of the dynamics of quantum phase transitions [1] fosters our understanding of the complex behavior of many-body systems far from equilibrium. This concerns, in particular, the interplay of the microscopic degrees of freedom, their entanglement, and the resulting emergent behavior. The controlled study of the time development of correlation functions expressing these entanglement properties, and the dynamical emergence of correlations from an initially uncorrelated state has been undertaken for various systems. The Bose-Hubbard model, describing the essential archetype of an emergence of strong correlations when one crosses a quantum phase transition point, was originally introduced in a conventional condensed matter context, to explain certain properties of bosons in periodic and/or random potentials [2]. Its implementation with ultracold atoms [3] and the subsequent experimental realization of the superfluid-Mott transition [4] has caused a flurry of research activity.

This activity is reviewed from a theoretical point of view in [5], while a number of recent experimental efforts studying ultracold atoms in optical lattices are covered, e.g., in [6]. Initially, theoretical studies were primarily devoted to the transition from the Mott regime, where number fluctuations are frozen (for commensurate filling of the lattice sites), to the superfluid side of the transition for which, conversely, phase fluctuations are frozen [7-11]. Most of these investigations were done numerically, with the exception of certain exactly solvable cases like the Ising chain in a transverse field [12].

We discuss here in detail a mean-field approach, first presented in [13], which enables the (in some particular cases analytical) rigorously controlled calculation of quantum correlations developing in rapid quenches from the superfluid to the Mott insulating phase. Such a number-conserving and hence controlled mean-field approach is valid at large filling $n \gg 1$ of the lattice sites, with the square root of the inverse filling $1 / \sqrt{n}$ providing the expansion parameter [14]. Here, we supply in particular, analytical estimates for the applica- bility of the results obtained in [13] to the experimentally relevant harmonically trapped case, by comparing the decay time of superfluid coherence to the propagation of the disturbances in the system induced by the quench. It should be noted that the mean-field "hydrodynamic" limit of large site fillings considered in the following is not of purely academic interest. While many experiments on the Mott transition are carried out at small filling of order unity, experiments on number squeezing at large filling have indeed been performed as well [15-17], already on an early stage of research into the possible occurrence of the Mott insulator transition [15].

\section{BOSE-HUBBARD MODEL AT LARGE FILLING}

The Bose-Hubbard model describes interacting bosons on a lattice, hopping from site to site. Restricting ourselves to (two-body) contact interactions, i.e., that two bosons interact only if at the same site $\alpha$, and to one single-particle state at each site, adding a one-particle scalar potential term, the Hamiltonian reads

$$
\hat{H}=J \sum_{\alpha \beta} M_{\alpha \beta} \hat{a}_{\alpha}^{\dagger} \hat{a}_{\beta}+\frac{U}{2} \sum_{\alpha}\left(\hat{a}_{\alpha}^{\dagger}\right)^{2} \hat{a}_{\alpha}^{2}+\sum_{\alpha} V_{\alpha} \hat{n}_{\alpha}
$$

The coupling constant $U$ is linear in the bulk contact interaction coupling constant $g$. The externally imposed scalar potential to additionally confine the atoms in the lattice is in most experimental situations to date to a good approximation harmonic $V_{\alpha} \propto \alpha^{2}$, or linear as in [17], $V_{\alpha} \propto \alpha$. The matrix $M_{\alpha \beta}$ describes the fact that, in the lattice, the effective mass (and possible higher terms in a gradient expansion), can in general depend on position and the direction of hopping of the particles from site to site. For the simplest example of a one-dimensional chain with nearest-neighbor hopping, we have $M_{\alpha \beta}=\delta_{\alpha, \beta}-\frac{1}{4}\left(\delta_{\alpha, \beta+1}+\delta_{\alpha, \beta-1}+\delta_{\alpha-1, \beta}+\delta_{\alpha+1, \beta}\right)$, and the effective mass of the bosons is given by $1 / m^{*}=J a^{2}$, where $a$ is the lattice spacing.

At large (average) filling $n \gg 1$ the Bose-Hubbard Hamiltonian (1) can be mapped to the so-called quantum rotor 
model (cf., e.g., $[1,18])$. Insertion of the (quantum) Madelung transformation $\hat{a}_{\alpha}=e^{i \hat{\phi}_{\alpha}} \sqrt{\hat{n}_{\alpha}}$ into Eq. (1) and expansion into inverse powers of $n$ yields the quantum rotor Hamiltonian

$$
\begin{aligned}
\hat{H} & =\sum_{\alpha}\left\{-n J \cos \left[\hat{\phi}_{\alpha}-\hat{\phi}_{\alpha+1}\right]+\frac{U}{2}\left(\hat{n}_{\alpha}-n\right)^{2}\right\} \\
& =\sum_{\alpha}\left\{-n J \cos \left[\hat{\phi}_{\alpha}-\hat{\phi}_{\alpha+1}\right]-\frac{U}{2} \frac{\partial^{2}}{\partial \hat{\phi}_{\alpha}^{2}}\right\},
\end{aligned}
$$

where we have taken for simplicity a one-dimensional (1D) lattice with no external trapping, $V_{\alpha}=0$. As an important ingredient, we use in this representation that local number fluctuation $\delta \hat{n}_{\alpha}=\hat{n}_{\alpha}-n$ and phase variables are conjugate in the limit of large $n$,

$$
\left[\hat{\phi}_{\alpha}, \delta \hat{n}_{\beta}\right]=i \delta_{\alpha \beta} .
$$

The fact that the number and phase variables are canonically conjugate variables (implying the very existence of a phase operator) depends on applying the mean-field (and effectively hydrodynamic, i.e., coarse-grained) limit $n \rightarrow \infty$ has been known for a long time (cf., e.g., [19,20]). The problems arising without the limit $n \rightarrow \infty$ can be seen by means of the full commutator

$$
\left[\hat{\phi}_{\alpha}, \hat{n}_{\beta}\right] \stackrel{?}{=} i \delta_{\alpha \beta} .
$$

Since $\hat{n}_{\beta}$ possesses a discrete spectrum with proper eigenvectors $\left|n_{\alpha}\right\rangle$, taking the $\left|n_{\alpha}\right\rangle$-expectation value of the above relation yields for $\alpha=\beta$ a contradiction. In the large $n$ limit, however, the normalized fluctuations $\delta \hat{n}_{\alpha} / n$ have quasicontinuous spectra and in this sense Eq. (3) provides a valid effective description.

The form (2) provides a nice and intuitive understanding of the two phases: In the superfluid state, the first term on the right-hand side dominates and $\hat{\phi}_{\alpha}-\hat{\phi}_{\alpha+1}$ is small (phase coherence). In the Mott phase, on the other hand, the second term wins and the number fluctuations $\delta \hat{n}_{\alpha}$ become small. Therefore, we may directly read off the scaling of the critical point $J_{c}$, which separates the two regimes $J_{c}=O(U / n)$. We depict a schematic representation of the superfluid-Mott transition on a quadratic two-dimensional lattice in Fig. 1.

\section{DYNAMICAL MEAN-FIELD THEORY}

We first consider the case without external trapping $V_{\alpha}$ $=0$, for which the filling is homogeneous $\left\langle\hat{n}_{\alpha}\right\rangle=n$. From Eq. (1), the Heisenberg equations of motion for the lattice field operator are (we take $\hbar \equiv 1$ throughout)

$$
i \partial_{t} \hat{a}_{\alpha}=J \sum_{\beta} M_{\alpha \beta} \hat{a}_{\beta}+U \hat{n}_{\alpha} \hat{a}_{\alpha} .
$$

To proceed, we now employ a lattice version of the numberconserving mean-field expansion which in its continuum version has been introduced in $[21,22]$. Since the Hamiltonian (1) cannot be diagonalized exactly, a controlled analytical approach requires a small or large parameter such that we
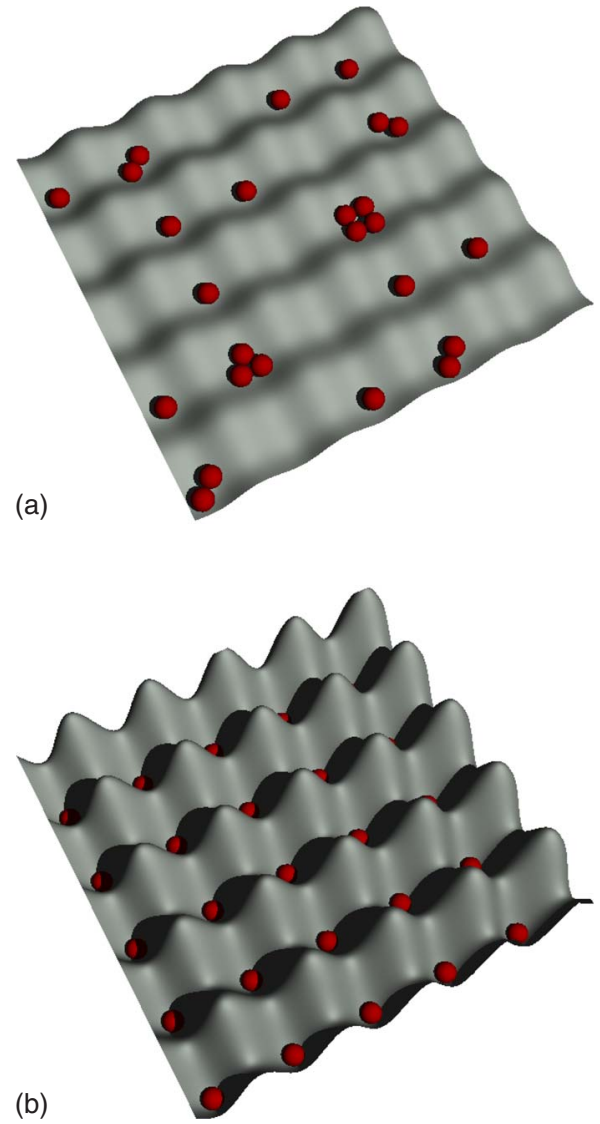

FIG. 1. (Color online) Localization (superfluid-Mott) transition of particles in an optical lattice. For increasing lattice depth and commensurate filling (integer number of particles per site), the system performs a quantum phase transition from a superfluid declocalized phase with highly mobile bosons (a) to a localized insulator phase with immobile bosons at commensurate filling (b). The present analysis investigates the temporal evolution of correlations when one is rapidly going from (a) [superfluid] to (b) [Mott]. For the sake of this representation, we display the non-mean-field case of unit filling, $n=1$, here.

can employ an expansion into powers of this control parameter. One option would be the weak-coupling regime $J \gg U$, where an expansion into powers of $U / J$ justifies the meanfield approach. However, since we want to study the sweep to the Mott phase, this ratio does not remain small and hence cannot be used as a control parameter. Therefore, we focus on the case of large fillings $n \gg 1$ here and use $1 / n$ as a small expansion parameter [23], i.e., in the limit of large fillings $n$, the full site field operator is expanded into terms of various power in $n$,

$$
\hat{a}_{\alpha}=\left(\psi_{0}+\hat{\chi}_{\alpha}+\hat{\zeta}_{\alpha}\right) \frac{\hat{A}}{\sqrt{\hat{N}}} .
$$

The operator $\hat{A}=\hat{a}_{\Sigma}\left(\hat{a}_{\Sigma}^{\dagger} \hat{a}_{\Sigma}\right)^{-1 / 2} \hat{N}^{1 / 2}$ with $\hat{a}_{\Sigma}=\Sigma_{\alpha} \hat{a}_{\alpha}$ accounts for the conservation of the total number of particles $\hat{N}$ $=\hat{A}^{\dagger} \hat{A}=\Sigma_{\alpha} \hat{n}_{\alpha}$. Due to the number-conserving nature of the ansatz, we have $\left\langle\hat{a}_{\alpha}\right\rangle=0$ exactly, as opposed to non-number- 
conserving approaches, in which $\left\langle\hat{a}_{\alpha}\right\rangle=O(\sqrt{n})$. We stress that a number-conserving formalism is essential to describe correlation functions accurately, especially those of higher order.

As stated before, the above mean-field expansion requires that, at each site, the integral filling $n_{\alpha}=\left\langle\hat{n}_{\alpha}\right\rangle=\left\langle\hat{a}_{\alpha}^{\dagger} \hat{a}_{\alpha}\right\rangle \in \mathbb{N}$ is much larger than unity, $n_{\alpha} \gg 1$. The idea of Eq. (6) is to expand the original operator $\hat{a}_{\alpha}$ into powers of $n_{\alpha} \gg 1$, considering the (formal) limit in which $n \uparrow \infty$ but with the chemical potential $\mu=U n$ remaining finite and fixed, such that $U$ $=O(1 / n)$. The leading term in the above expansion (6) is the order parameter $\psi_{0}=O(\sqrt{n})$ describing the condensate part. The linearized quantum corrections $\hat{\chi}_{\alpha}=O\left(n^{0}\right)$ are decomposed into single-particle contributions, and correspond to Bogoliubov quasiparticle excitations above the superfluid ground state, after a Bogoliubov transformation to a quasiparticle basis. For the validity of the expansion (6), the remaining (nonlinear) higher-order corrections $\hat{\zeta}_{\alpha}$ containing multiparticle contributions must remain small, $\hat{\zeta}_{\alpha} \ll 1$, during the whole temporal evolution of the system. Since we are dealing with formally unbounded operators, the condition $\hat{\zeta}_{\alpha} \ll 1$ should be understood as a weak limit, i.e., $\left\langle\hat{\zeta}_{\alpha}\right\rangle \ll 1$ and $\left\langle\hat{\zeta}_{\alpha}^{\dagger} \hat{\zeta}_{\alpha}\right\rangle \ll 1$, etc.

Thus, $\hat{\zeta}_{\alpha}$ of order unity signifies that one approaches strongly correlated states, i.e., the Mott regime. These "strong correlations" are due to interactions between the quasiparticle excitations $\hat{\chi}_{\alpha}$, which become stronger when approaching the transition, and eventually generate a gap due to nonperturbative effects. The emergence of this gap denotes the transition from the delocalized atoms (superfluid state) to the localized atoms (Mott state), see, e.g., [24]. In what follows, we are interested in describing the approach to the localized state of the atoms, and how in a dynamical quantum phase transition the corresponding decrease in the phase ordering takes place.

The Bogoliubov-de Gennes equations for the quasiparticle excitations at site $\alpha$, which are by definition described by the first-order operators $\hat{\chi}_{\alpha}$, read

$$
i \partial_{t} \hat{\chi}_{\alpha}=J \sum_{\beta} M_{\alpha \beta} \hat{\chi}_{\beta}+2 U\left|\psi_{0}^{2}\right| \hat{\chi}_{\alpha}+U \psi_{0}^{2} \hat{\chi}_{\alpha}^{\dagger},
$$

with $\psi_{0}$ being determined by the solution of the GrossPitaevskiı̌ mean-field equation

$$
i \partial_{t} \psi_{0}=J \sum_{\beta} M_{\alpha \beta} \psi_{0}+U\left|\psi_{0}\right|^{2} \psi_{0}=U\left|\psi_{0}\right|^{2} \psi_{0},
$$

where the final equality holds for a spatially homogeneous ground state. All the residual terms, remaining after the insertion of Eq. (6) into Eq. (5), determine the higher-order corrections $\hat{\zeta}_{\alpha}$ to the mean-field expansion (6), which then evolve according to the equation of motion

$$
\begin{aligned}
i \partial_{t} \hat{\zeta}_{\alpha}= & J \sum_{\beta} M_{\alpha \beta} \hat{\zeta}_{\beta}+2 U\left|\psi_{0}^{2}\right| \hat{\zeta}_{\alpha}+U \psi_{0}^{2} \hat{\zeta}_{\alpha}^{\dagger}+2 U \psi_{0} \hat{\chi}_{\alpha}^{\dagger} \hat{\chi}_{\alpha} \\
& +U \psi_{0}^{*} \hat{\chi}_{\alpha}^{2}+U \hat{\chi}_{\alpha}^{\dagger} \hat{\chi}_{\alpha}^{2}+O\left(U \hat{\zeta}_{\alpha}\right) .
\end{aligned}
$$

Deep in the superfluid phase (our initial state), the higher- order corrections $\hat{\zeta}_{\alpha}$ are small and the mean-field expansion (6) works very well. If we approach the Mott phase, however, these corrections start to grow according to Eq. (9) and, at some point, the mean-field expansion (6) breaks down. The characteristic time scale of this breakdown can be estimated from the nonlinear source terms in Eq. (9), which are suppressed to $O(1 / \sqrt{n})$ in view of the imposed constancy of the chemical potential, so that $U=O(1 / n), \psi_{0}=O(\sqrt{n})$, and $\hat{\chi}_{\alpha}=O\left(n^{0}\right)$. Therefore (starting in the superfluid phase), the higher-order corrections remain small as long as we have $U t \sqrt{n} \ll 1$, i.e., for evolution times which are of order $t$ $=O(\sqrt{n})$. Thus, while Bogoliubov mean-field theory is, in principle, also valid (initially, i.e., for $J \gg U$ ) for small values of $n=O(1)$, the $\hat{\zeta}_{\alpha}$ corrections in Eq. (9), which describe correlations beyond those derivable from the mean-field plus single-particle fluctuations, i.e., from $\psi_{0}$ and $\hat{\chi}_{\alpha}$ in Eq. (6), begin to grow very quickly in off-equilibrium situations, then invalidating the Bogoliubov approach at small filling. For large fillings $n \gg 1$, however, the mean-field expansion (6) can be extrapolated to relatively long time scales $t=O(\sqrt{n})$, which allows us to study the sweep analytically (with $\hat{\zeta}_{\alpha}$ serving as an indicator for the validity of the mean-field approach).

Linearizing the polar decomposition of the fundamental field operator $\hat{a}_{\alpha}=\exp \left\{i \hat{\phi}_{\alpha}\right\} \sqrt{\hat{n}_{\alpha}}$, we may identify the fluctuations $\hat{\chi}_{\alpha}=\psi_{0}\left[\delta \hat{n}_{\alpha} /(2 n)+i \delta \hat{\phi}_{\alpha}\right]+O(1 / \sqrt{n})$ in terms of the linearized number fluctuations $\delta \hat{n}_{\alpha}$ and the conjugate phase fluctuations $\delta \hat{\phi}_{\alpha}$ according to Eq. (3). Equation (7) can be diagonalized by a normal-mode expansion into the eigenvectors $M_{\alpha \beta} v_{\kappa}^{\beta}=\lambda_{\kappa} v_{\kappa}^{\alpha}$ of the hopping matrix $M_{\alpha \beta}$, with the eigenvalues $\lambda_{\kappa}$ labeled by the generalized momenta $\kappa$. To this end, we expand the number and phase fluctuations via $\delta \hat{n}_{\kappa}$ $=v_{\kappa}^{\alpha} \delta \hat{n}_{\alpha}$ and $\delta \hat{\phi}_{\kappa}=v_{\kappa}^{\alpha} \delta \hat{\phi}_{\alpha}$, respectively, and insert them into

$$
\hat{\chi}_{\alpha}=\sqrt{n} e^{-i n \int U d t}\left(\delta \hat{n}_{\alpha} / 2 n+i \delta \hat{\phi}_{\alpha}\right),
$$

where we have factored out the time dependence of the Gross-Pitaevskil wave function with the appropriate phase factor $\psi_{0}=\sqrt{n} e^{-i n \int U d t}$ as follows from Eq. (8). Thus, we obtain from the Bogoliubov-de Gennes equation (7), two real equations for the time evolution of density and phase fluctuations in the eigenbasis labeled by $\kappa$,

$$
\begin{gathered}
\partial_{t} \delta \hat{n}_{\kappa}=2 n J \lambda_{\kappa} \delta \hat{\phi}_{\kappa}, \\
\partial_{t} \delta \hat{\phi}_{\kappa}=-\left(\frac{J \lambda_{\kappa}}{2 n}+U\right) \delta \hat{n}_{\kappa},
\end{gathered}
$$

for generally time-dependent $J$ and $U$. In case that both $J$ and $U$ are constant in time, the above equations result in the well-known Bogoliubov spectrum $[25,26]$

$$
\omega_{\kappa}^{2}=J^{2} \lambda_{\kappa}^{2}+2 n U J \lambda_{\kappa} .
$$

We now discuss two possible routes to approach or cross the phase transition from the superfluid to the Mott side dynamically. Either one decreases the tunneling rate in time or, alternatively, the interaction is increased to suppress the superfluid density at given filling and thus cross the transition line. 
TABLE I. A few analytically solvable cases for time-dependent $J=J(t)$. The last column indicates the behavior of the argument $x_{\kappa}$ at late laboratory time.

\begin{tabular}{ccccc}
\hline \hline \multicolumn{1}{c}{ Solution } & Argument & Indices and constants & Asymptotics $t \uparrow \infty$ \\
\hline$J_{0} e^{-\gamma t}$ & $W_{i \nu, \mu}\left(2 i x_{\kappa}\right)$ & $x_{\kappa}=\tau_{\kappa}=-J_{0} \lambda_{\kappa} e^{-\gamma t} / \gamma$ & $\mu=1 / 2, \nu=U n / \gamma$ & $x_{\kappa} \uparrow 0$ \\
$J_{0}(\gamma t)^{-2}$ & $\sqrt{x_{\kappa}} H_{\nu}^{(1)}\left(x_{\kappa}\right)$ & $x_{\kappa}=\tau_{\kappa}=-J_{0} \lambda_{\kappa}(\gamma t)^{-1 / \gamma}$ & $\nu=\sqrt{1 / 4-2 U n J_{0} \lambda_{\kappa} / \gamma^{2}}$ & $x_{\kappa} \uparrow 0$ \\
$J_{0}(\gamma t)^{-1}$ & $H_{\nu}^{(1)}\left(x_{\kappa}\right)$ & $x_{\kappa}=2 \sqrt{2 U n J_{0} \lambda_{\kappa}}(\gamma t)^{1 / 2} / \gamma$ & $\nu= \pm 2 i J_{0} \lambda_{\kappa} / \gamma$ & $x_{\kappa} \uparrow \infty$ \\
$J_{0}(\gamma t)^{-2 / 3}$ & $x_{\kappa}^{-1 / 4} W_{i \nu, \mu}\left(i x_{\kappa}\right)$ & $x_{\kappa}=3 \sqrt{2 U n J_{0} \lambda_{\kappa}}(\gamma t)^{2 / 3} / \gamma$ & $\mu^{2}=1 / 16, \nu^{2}=9\left(J_{0} \lambda_{\kappa}\right)^{3} /\left(32 U n \gamma^{2}\right)$ & $x_{\kappa} \uparrow \infty$ \\
$J_{0}(\gamma t)^{-1 / 2}$ & $\sqrt{x_{\kappa}} H_{\nu}^{(1)}\left(c_{\kappa} x_{\kappa}^{3 / 2}\right)$ & $x_{\kappa}=\sqrt{\gamma t}+J_{0} \lambda_{\kappa} /(2 U n)$ & $\nu=1 / 3, c_{\kappa}=\sqrt{2 U n J_{0} \lambda_{\kappa}} /(3 \gamma)$ & $x_{\kappa} \uparrow \infty$ \\
\hline \hline
\end{tabular}

It is demonstrated that in several particular cases for $J=J(t)$ or $U=U(t)$, respectively, the Bogoliubov-de Gennes equations (7) can be solved analytically.

\section{A. Decreasing the tunneling rate}

Combining the two equations (11), we get the following second-order equation for the number fluctuations [analogously for phase fluctuations, see Eq. (26) below]

$$
\left(\frac{\partial}{\partial t} \frac{1}{J} \frac{\partial}{\partial t}+\lambda_{\kappa}\left[J \lambda_{\kappa}+2 U n\right]\right) \delta \hat{n}_{\kappa}=0 .
$$

Dividing this equation by $J$ and $\lambda_{\kappa}^{2}$, and defining a new time coordinate depending on the mode index $\kappa$,

$$
d \tau_{\kappa}=\lambda_{\kappa} J d t,
$$

we obtain an equation containing the operator $\partial^{2} / \partial \tau_{\kappa}^{2}$,

$$
\left(\frac{\partial^{2}}{\partial \tau_{\kappa}^{2}}+1+\frac{U}{J} \frac{2 n}{\lambda_{\kappa}}\right) \delta \hat{n}_{\kappa}=0 .
$$

We note that this equation now contains the ratio $U / J$ only, but not $U$ and $J$ separately (cf. Sec. III C below).

Let us first study the case $J=J(t)$ while $U=$ const. Even though it is not possible to give a closed solution of Eq. (13) for arbitrary time dependences $J(t)$, there are several cases which do admit analytic expressions in terms of Hankel $H_{\nu}^{(1)}$ or Whittaker $W_{\nu, \mu}$ functions [31]. We listed a few cases in Table I.

In view of the asymptotic $t \uparrow \infty$ behavior of the Hankel and Whittaker functions [31], we see that the number fluctuations $\delta \hat{n}_{\kappa}$ oscillate forever in the last three cases $J(t)$ $\propto t^{-1}, t^{-2 / 3}, t^{-1 / 2}$, though with a decreasing amplitude and frequency. In the case of an exponential-i.e., much fastersweep $J(t)=J_{0} e^{-\gamma t}$, on the other hand, the solutions $\delta \hat{n}_{\kappa}$ do not have enough time to adjust to the externally imposed change of $J(t)$ and freeze at a finite value (nonadiabatic behavior). Finally, the second case $J(t)=J_{0}(\gamma t)^{-2}$ just marks the border between the two regimes (eternal oscillation versus freezing). Consequently, the number fluctuations $\delta \hat{n}_{\kappa}$ vanish for late times in this situation (for $\lambda_{\kappa}>0$ ).

The asymptotic behavior can be interpreted nicely in terms of the analogy to cosmology sketched in Sec. V below.
The freezing of the number fluctuations $\delta \hat{n}_{\kappa}$ for $J(t)=J_{0} e^{-\gamma t}$ can then be understood via the emergence of a horizon analog-whereas for $J(t) \propto t^{-1}, t^{-2 / 3}, t^{-1 / 2}$, such a horizon is absent. The critical behavior $J(t)=J_{0}(\gamma t)^{-2}$ precisely marks the limit for horizon formation (cf. Sec. V).

It is very illustrative to compare the various cases discussed above to a harmonic oscillator with a time-dependent damping term and/or spring constant. In the last three cases $J(t) \propto t^{-1}, t^{-2 / 3}, t^{-1 / 2}$, the spring constant dominates (underdamped oscillator) while the exponential sweep $J(t)=J_{0} e^{-\gamma t}$ induces a transition to the overdamped regime at some time. The boundary case $J(t)=J_{0}(\gamma t)^{-2}$ would then correspond to critical damping, where the solution $\delta \hat{n}_{\kappa}$ approaches zero very quickly. For the Bose-Hubbard model, this time dependence would allow us to approach the Mott state very efficiently.

However, in an experimental realization, a dynamics $J(t)=J_{0}(\gamma t)^{-2}$ requires some fine tuning and is probably hard to achieve. The exponential sweep is more interesting from a theory point of view (since it yields nonzero frozen number fluctuations) and should also be a better approximation to a realistic experimental situation. The experimental relevance becomes apparent considering the fact that, in sufficiently deep $d$-dimensional simple cubic lattices, $J(t)$ $\propto\left(V_{0}(t) / E_{R}\right)^{3 / 4} \exp \left\{-2 \sqrt{V_{0}(t) / E_{R}}\right\}$ and $U \propto\left(V_{0} / E_{R}\right)^{d / 4}$ hold, where $V_{0}(t)$ is the time-dependent lattice depth given by the laser intensity and $E_{R}=\pi^{2} /\left(2 m a^{2}\right)$ is the (constant) recoil energy, with $m$ the boson mass [32]. Apart from logarithmically slow corrections, an exponential sweep $J(t) \propto e^{-\gamma t}$ therefore corresponds to increasing the laser amplitude (and therefore, $\sqrt{V_{0}}$ ) linearly in time, with $U$ then remaining approximately constant.

For the case of an exponentially decreasing tunneling rate, a universal "scaling" solution exists: As we may infer from Table I, the solution (i.e., the indices $\mu$ and $\nu$ of the Whittaker function $\left.W_{\nu, \mu}\right)$ then do not depend on $\kappa$. The scaling time from Eq. (14), depending on the mode index $\kappa$, reads $\tau_{\kappa}=-\lambda_{\kappa} J(t) / \gamma$, so that Eq. (13) is transformed into a scale invariant equation of the form of Eq. (15),

$$
\left(\frac{\partial^{2}}{\partial \tau_{\kappa}^{2}}+\left[1-\frac{2}{\tau_{\kappa}} \frac{U n}{\gamma}\right]\right) \delta \hat{n}_{\kappa}=0 .
$$

The only remaining dimensionless parameter determining the relevant universality class of solutions of this equation is 
$\nu=U n / \gamma$. This parameter, equal to the ratio of chemical potential $\mu=U n$ (i.e., internal energy scale) and sweep rate $\gamma$ (i.e., external time scale), represents a measure of the rapidity of the externally imposed sweep: $\nu \gg 1$ implies a slow and $\nu \ll 1$ a fast (nonadiabatic) sweep. The adiabaticity parameter $\nu$ determines the nature of the final state. Starting in the superfluid phase and ramping down the tunneling rate very rapidly, $\nu \ll 1$, the system will have no time to react to this change. For later times, the vanishing hopping $J=0$ prevents an equilibration of the number fluctuations, i.e., they will be as large as in the initial coherent state in this situation. The slower we sweep $J(t)$, the more time the system has to adapt to this external change and the closer the final state will be to the Mott state (i.e., smaller number fluctuations will result from a slow sweep).

As listed in Table I, the analytical solution of Eq. (16) can be found in terms of the Whittaker functions $W_{i \nu, 1 / 2}$ [31]. It allows us to obtain the following exact Bogoliubov transformation from bare density or filling fluctuation to initial vacuum quasiparticle operators $\hat{b}_{\kappa}$, labeled by the mode in$\operatorname{dex} \kappa$,

$$
\delta \hat{n}_{\kappa}=\sqrt{n} e^{-\pi \nu / 2} W_{i \nu, 1 / 2}\left(2 i \tau_{\kappa}\right) \hat{b}_{\kappa}+\text { H.c. }
$$

The initial vacuum quasiparticle operators $\hat{b}_{\kappa}$ annihilate the adiabatic superfluid ground state $\hat{b}_{\kappa}|\mathrm{in}\rangle=0$ at early times $\tau_{\kappa} \downarrow-\infty$, where the modes oscillate like $e^{ \pm i \tau_{\kappa}}$. (Note that the scaling time $\tau_{\kappa}$ is dimensionless.) The phase fluctuations are obtained using the relation $\delta \hat{\phi}_{\kappa}=-\frac{1}{2 n} d \delta \hat{n}_{\kappa} / d \tau_{\kappa}$ following from Eqs. (11) and (14),

$$
\delta \hat{\phi}_{\kappa}=-\frac{e^{-\pi \nu / 2}}{2 \sqrt{n}} \frac{d W_{i \nu, 1 / 2}\left(2 i \tau_{\kappa}\right)}{d \tau_{\kappa}} \hat{b}_{\kappa}+\text { H.c. }
$$

The analytical scaling solution thus obtained represents, to the best of our knowledge, the first example of an exact solution of the (nonintegrable) Bose-Hubbard model on the Bogoliubov mean-field level in a dynamical situation.

Due to the perfect scaling solution in Eq. (17), the frozen value of the number fluctuations at late times, when $\tau_{\kappa} \rightarrow 0$, is independent of $\kappa$, but the decaying corrections do depend on the eigenvalue $\lambda_{\kappa}$ as follows:

$$
\left\langle\delta \hat{n}_{\kappa}^{2}\right\rangle \equiv\left\langle\operatorname{in}\left|\left(\delta \hat{n}_{\kappa}\right)^{2}\right| \text { in }\right\rangle=n \frac{1-e^{-2 \pi \nu}}{2 \pi \nu}+O\left(t \lambda_{\kappa} e^{-\gamma t}\right) .
$$

Since the leading term is independent of $\kappa$, it just yields a local $\left(\propto \delta_{\alpha, \beta}\right)$ contribution after the mode sum $(\kappa \rightarrow \alpha)$ and thus leads to frozen on-site number variations.

In contrast to the number fluctuations which freeze according to Eq. (19), the conjugate phase fluctuations grow (as one would expect when approaching the Mott phase). From our analytical result (18), we conclude that they increase (initially) quadratically in time,

$$
\left\langle\delta \hat{\phi}_{\kappa}^{2}\right\rangle=\nu \frac{1-e^{-2 \pi \nu}}{2 \pi n} \gamma^{2} t^{2}+O\left(\gamma t \ln \lambda_{\kappa}\right) .
$$

Again, like for the number fluctuations, the leading (first) term is independent of the mode index $\kappa$ and yields the on- site phase fluctuations $\left\langle\delta \hat{\phi}_{\alpha}^{2}\right\rangle$. The off-site phase correlations $\left\langle\delta \hat{\phi}_{\alpha} \delta \hat{\phi}_{\beta}\right\rangle$, corresponding to the second term, grow linearly in time (initially).

\section{B. Final state}

All the results so far were obtained by a controlled extrapolation of the mean-field expansion (6) from the weakcoupling ( $J$ dominates) into the strong-coupling regime $(U$ dominates) and hence are only valid as long as the quantum depletion $\left\langle\hat{\chi}_{\alpha}^{\dagger} \hat{\chi}_{\alpha}\right\rangle$ is small, i.e., the condensate $\psi_{0}$ dominates. In the strong-coupling regime, however, the quantum depletion grows (on a time scale of order $\sqrt{n}$ ) and finally invalidates the mean-field expansion (6). Fortunately, we may also analytically describe the ensuing stages of the quantum evolution, because the tunneling rate $J(t \gg 1 / \gamma) \lll 1$ is exponentially small and can be completely neglected. In this completely interaction-dominated limit, the evolution of the site operators can be approximated by $d \hat{a}_{\alpha} / d t=-i U \hat{n}_{\alpha} \hat{a}_{\alpha}$, which possesses the simple exponential solution

$$
\hat{a}_{\alpha}(t)=\exp \left\{-i U \hat{n}_{\alpha}^{0} t\right\} \hat{a}_{\alpha}^{0} \quad(J \lll 1) .
$$

Consequently, we may calculate the time evolution of correlation functions throughout the dynamics (i.e., for all $t$ ) by using the results of the mean-field expansion, which are valid for intermediate times with $\gamma t \gg 1$ and $U t \sqrt{n} \ll 1$, as initial conditions, and then switching to the above solution (21) for later stages.

A frequently used indicator for distinguishing the superfluid from the Mott phase is the off-diagonal long-range order (ODLRO) usually associated to the first-order correlation function $\left\langle\hat{a}_{\alpha}^{\dagger}(t) \hat{a}_{\beta}(t)\right\rangle$. Using the results above, we may derive an analytical expression for the time dependence of $\left\langle\hat{a}_{\alpha}^{\dagger}(t) \hat{a}_{\beta}(t)\right\rangle$ during and after the sweep. From Eq. (21), we obtain for the correlator

$$
\left\langle\hat{a}_{\alpha}^{\dagger}(t) \hat{a}_{\beta}(t)\right\rangle=n\left\langle\exp \left\{i U\left(\hat{n}_{\alpha}-\hat{n}_{\beta}\right) t\right\}\right\rangle+O(\sqrt{n}) .
$$

On the other hand, the frozen first-order number fluctuations $\delta \hat{n}_{\alpha}$ are then in a squeezed state which can (for $n \gg 1$ ) be approximated by a (continuous) Gaussian distribution. For a Gaussian variable $X$ with $\langle X\rangle=0$, the exponential average yields $\langle\exp \{i X\}\rangle=\exp \left\{-\left\langle X^{2}\right\rangle / 2\right\}$ and hence, we finally get

$$
\left\langle\hat{a}_{\alpha}^{\dagger}(t) \hat{a}_{\beta}(t)\right\rangle \approx n \exp \left\{-U^{2} t^{2} \Delta^{2}\left(n_{\alpha}\right)\right\},
$$

where the on-site number variations are given by Eq. (19),

$$
\Delta^{2}\left(n_{\alpha}\right)=\left\langle\hat{n}_{\alpha}^{2}\right\rangle-\left\langle\hat{n}_{\alpha}\right\rangle^{2}=\left\langle\delta \hat{n}_{\alpha}^{2}\right\rangle=n \frac{1-e^{-2 \pi \nu}}{2 \pi \nu} .
$$

The result (23) represents an extension to the present period lattice geometry of the result obtained in [28], where the interaction-induced decay of coherence in a double-well trap was studied. The Fourier transform of the first-order correlation function $\left\langle\hat{a}_{\alpha}^{\dagger}(t) \hat{a}_{\beta}(t)\right\rangle$ determines the corresponding momentum distribution function $g_{1}(\boldsymbol{k})$. The decay of the offdiagonal long-range order (ODLRO) in Eq. (23) thus directly corresponds to a temporal decrease of the peak in $g_{1}(\boldsymbol{k})$ at $k=0$, measurable in time-of-flight experiments $[29,30]$.

In summary, the state obtained with $J(t \gg 1 / \gamma) \lll 1$ (while still maintaining $U t \sqrt{n} \ll 1$, i.e., at intermediate times), 
TABLE II. Important dual cases for temporal variations of $U$ and $J$. Given the solution for the first column, we can immediately conclude, by a simple time transformation, on the solution for the second column, and vice versa.

\begin{tabular}{ccc}
\hline \hline$J(t)$ & Dual $U(t)$ & Dual Range \\
\hline$J_{0} e^{-\gamma t}$ & $U_{0}(\gamma t)^{-1}$ & $-\infty<t<0$ \\
$J_{0}(\gamma t)^{-2}$ & $U_{0}(\gamma t)^{-2}$ & $-\infty<t<0$ \\
$J_{0}(\gamma t)^{-1}$ & $U_{0} e^{\gamma t}$ & $-\infty<t<\infty$ \\
$J_{0}(\gamma t)^{-2 / 3}$ & $U_{0}(\gamma t)^{2}$ & $0<t<\infty$ \\
$J_{0}(\gamma t)^{-1 / 2}$ & $U_{0} \gamma t$ & $0<t<\infty$ \\
\hline
\end{tabular}

which has frozen number fluctuations according to Eq. (19), forms an appropriate initial many-body quantum state for the further evolution beyond mean-field into the strongly correlated Mott phase.

\section{Increasing the interaction coupling}

Another possibility to approach the Mott phase dynamically is to increase $U$ in time. This can be experimentally realized using a time-dependent sweep through a Feshbach resonance, which varies the $s$-wave scattering length $a_{s}$, and thus $U$ only, while keeping $V_{0}$ and thus $J$ fixed. Since Eq. (15) depends on the ratio $U / J$ only, increasing $U$ is analogous to decreasing $J$. Therefore, we can establish an exact "duality" between the results obtained for $J=J(t)$ and the present $U=U(t)$, i.e., every analytic solution in Table I of Sec. III A corresponds to a dual expression for $U=U(t)$ after incorporating the transformation $t \rightarrow \tau$ of the time coordinate in Eq. (14). For a power-law dependence $U \propto t^{\alpha}$, Eq. (15) possesses formally the same solution as for $J \propto \tau^{-\alpha}$. Transforming back to the laboratory time via Eq. (14), this corresponds to $J \propto t^{-\alpha /(\alpha+1)}$ while the two limiting cases $\alpha=-1$ and $\alpha=\infty$ correspond to exponential dependences (see Table II). Due to the transformation $t \rightarrow \tau$ of the time coordinate in Eq. (14), the solutions for $U(t)$ do not freeze in terms of the laboratory time $t \rightarrow \infty$.

Again, it is likely that most of the dynamics $U(t)$ are hard to realize experimentally. Therefore, we focus on the linear increase $U(t)=\gamma t$ in the following, since this case is probably close to an experimental setup. For a linear growth [dually corresponding to $J(t) \propto 1 / \sqrt{t}]$, we can define a scaling time using a simple $\kappa$-dependent shift of the time origin,

$$
\tau_{\kappa}=t+\frac{J \lambda_{\kappa}}{2 n \gamma} \Leftrightarrow d \tau_{\kappa}=d t
$$

Introducing this scaling time into the equation for phase fluctuations

$$
\left(\frac{\partial}{\partial t} \frac{1}{2 n U(t)+J \lambda_{\kappa}} \frac{\partial}{\partial t}+J \lambda_{\kappa}\right) \delta \hat{\phi}_{\kappa}=0,
$$

we obtain a scaling equation of the following form:

$$
\left(\frac{\partial}{\partial \tau_{\kappa}} \frac{1}{\tau_{\kappa}} \frac{\partial}{\partial \tau_{\kappa}}+2 n \gamma J \lambda_{\kappa}\right) \delta \hat{\phi}_{\kappa}=0
$$

The solution of this equation can be found in terms of Hankel functions and leads us to the following Bogoliubov transformation for the phase fluctuations:

$$
\delta \hat{\phi}_{\kappa}=\hat{C}_{\kappa} \tau_{\kappa} H_{2 / 3}^{(1)}\left(\frac{2}{3} \sqrt{2 n \gamma J \lambda_{\kappa}} \tau_{\kappa}^{3 / 2}\right)+\text { H.c. }
$$

where $H_{\nu}^{(1)}$ and $H_{\nu}^{(2)}=\left(H_{\nu}^{(1)}\right)^{*}$ are Hankel functions of the first and second kind, respectively. Note that the index is mode number $\kappa$ independent, like the solution for exponential decrease of $J$ in Eq. (17). In contrast to the above-discussed case of an exponential decay of $J$, approaching the "hardcore" limit by letting $U$ grow (linearly), we do not obtain frozen number fluctuations at late times $\tau_{\kappa} \uparrow \infty$. We then have, instead, increasingly rapid oscillations of fluctuating filling

$$
\delta \hat{n}_{\kappa}=-\frac{1}{\gamma \tau_{\kappa}} \frac{d \delta \phi_{\kappa}}{d \tau_{\kappa}}=\hat{\widetilde{C}}_{\kappa} \tau_{\kappa}^{1 / 2} H_{1 / 3}^{(1)}\left(\frac{2}{3} \sqrt{2 n \gamma J \lambda_{\kappa}} \tau_{\kappa}^{3 / 2}\right)+\text { H.c. }
$$

where the operators $\hat{\widetilde{C}}_{\kappa}$ are proportional to the $\hat{C}_{\kappa}$ in Eq. (28). Due to the asymptotic (large $x$ ) behavior $H_{\nu}(x)$ $\rightarrow \sqrt{2 / \pi x} \exp [i(x-\nu \pi / 2-\pi / 4)]$, we have at late times $\delta \hat{n}_{\kappa}$ $\propto t^{-1 / 4} \exp \left[i \frac{2}{3} \sqrt{2 n \gamma J \lambda_{\kappa}} t^{3 / 2}\right]+$ H.c. From Eq (28), we derive the corresponding slow increase of the amplitude of phase fluctuations, $\delta \hat{\phi}_{\kappa} \propto t^{1 / 4} \exp \left[i \frac{2}{3} \sqrt{2 n \gamma J \lambda_{\kappa}} t^{3 / 2}\right]+$ H.c., with the same increase of the oscillation frequency.

\section{Quantum depletion}

Approaching the Mott phase entails that the condensate becomes depleted due to Bogoliubov quasiparticle excitations created above the (still superfluid) ground state. We stress that the quantum depletion we calculate below explicitly depends on $J=J(t)$ [or $U=U(t)]$ in a particular manner. Thus it is possible, within our controlled mean-field scheme, to unambiguously identify the number squeezing (related to the depletion) caused by the creation of the excitations, and to distinguish it from effects coming from the time dependence of the mean field in the Gross-Pitaevskil equation (which were discussed in [38]).

The (relative) depletion is defined by the expression

$$
\mathcal{D} \equiv \frac{\sum_{\alpha}\left\langle\hat{\chi}_{\alpha}^{\dagger} \hat{\chi}_{\alpha}\right\rangle}{\sum_{\alpha} n_{\alpha}} .
$$

Concentrating here on $J=J_{0} \exp [-\gamma t]$, where as shown in Sec. III A number fluctuations freeze, and using that the local depletion is given by $\hat{\chi}_{\alpha}^{\dagger} \hat{\chi}_{\alpha}=\delta \hat{n}_{\alpha}^{2} /(4 n)+n \delta \hat{\phi}_{\alpha}^{2}+1 / 2$, we have from Eqs. (19) and (20), 


$$
\begin{aligned}
\mathcal{D} & =\frac{\left\langle\hat{\chi}_{\alpha}^{\dagger} \hat{\chi}_{\alpha}\right\rangle}{n} \simeq \frac{1}{2 \pi n}\left(\nu \gamma^{2} t^{2}+\frac{1}{\nu}\right)\left(1-e^{-2 \pi \nu}\right)+\frac{1}{2 n} \\
& \simeq\left\{\begin{array}{ll}
U^{2} n t^{2} & \nu \ll 1 \\
\frac{U \gamma t^{2}}{2 \pi} & \nu \gg 1
\end{array},\right.
\end{aligned}
$$

which is valid provided that the mean-field condition $U t \sqrt{n}$ $\ll 1$ holds-which implies $\mathcal{D} \ll 1$. The phase fluctuations caused by the sweep always dominate the number fluctuations in the limit assumed throughout, $n \gg 1$ (while still keeping $U t \sqrt{n} \ll 1)$.

\section{E. Comparison to continuum scaling}

Up to now we have treated the homogeneous case. If the lattice is embedded in an external harmonic trapping potential, like in experiment, the derived analytical solutions for $J \propto \exp [-\gamma t]$ and $U \propto t$ are not exactly valid anymore. However, in the continuum limit, the well-known scaling approach for a bulk gas in a harmonic trapping potential [33-35] can be applied and it is rather interesting to contrast this approach with our scaling solutions on the lattice (17) and (29).

The continuum scaling equations of motion in $1 \mathrm{D}$ read

$$
\ddot{b}(t)+\frac{J(t)}{J_{0}} \omega_{0}^{2} b(t)-\frac{\dot{J}(t)}{J(t)} \dot{b}(t)=\frac{J(t)}{J_{0}} \frac{U(t)}{U_{0}} \frac{\omega_{0}^{2}}{b^{2}(t)},
$$

where $b(t)$ denotes the time-dependent scaling parameter [33-35] while $\omega_{0}, J_{0}$, and $U_{0}$ are the initial values for trap frequency, tunneling, and interaction rate, respectively. We conclude from Eq. (32) that the temporal change of $J(t)$ corresponds to a change in the (inverse) effective mass of the bosons.

For $J(t)=J_{0} e^{-\gamma t}$, the effective harmonic trapping frequency becomes exponentially slower, and the scaling factor motion consequently overdamped due to the resulting constant (Ohmic) damping. This damping corresponds to the derived freezing of the number fluctuations and increase of phase fluctuations on the lattice as follows:

$$
\ddot{b}(t)+\gamma \dot{b}(t)+e^{-\gamma t} \omega_{0}^{2} b(t)=\frac{e^{-\gamma t} \omega_{0}^{2}}{b^{2}(t)} .
$$

Conversely, for $U(t)$ linearly increasing in time, and constant $J$, due to the driving term on the right-hand side of Eq. (32), the scaling factor begins to oscillate increasingly fast, as already observed from the exact solution on the lattice, Eq. (29).

\section{NONEQUILIBRIUM QUANTUM EFFECTS IN INHOMOGENEOUS SYSTEMS}

In the additional presence of an optical lattice, the behavior of the system depends on the relation between the inhomogeneity of the trap potential $V_{\alpha}$ and the central filling $n_{\text {center }} \gg 1$. If the potential $V_{\alpha}$ is rather shallow, the system develops a "wedding cake" structure near the boundaries, where the filling is small and hence the Mott phase emerges
$[3,36]$. For stronger inhomogeneities of $V_{\alpha}$ and/or larger central fillings $n_{\text {center }} \gg 1$, on the other hand, two-body interactions $U\left(\hat{a}_{\alpha}^{\dagger}\right)^{2} \hat{a}_{\alpha}^{2}$ in Eq. (1) can be neglected in comparison with the tunneling term $J M_{\alpha \beta} \hat{a}_{\alpha}^{\dagger} \hat{a}_{\beta}$ and the potential gradient of $V_{\alpha} \hat{n}_{\alpha}$ due to $U=O\left(1 / n_{\text {center }}\right)$. Intuitively speaking, the various rims of the wedding cake would be compressed into a single lattice site and hence, disappear.

Lowering $J$ toward the (first) phase transition point, the wedding cake structure gradually develops by Mott insulator shells propagating inwards from the outer low density edges of the gas cloud. Therefore, we have to study the question of whether the results derived above for the homogeneous case-such as the temporal decay of the coherence peak of the momentum distribution function $g_{1}(\boldsymbol{k})$ due to number squeezing effects - can still be observed in the presence of an external trap. Clearly, near the boundaries inhomogeneity effects will be important. However, in the central region, the homogeneity assumption should provide a good approximation-provided that the momentum distribution function $g_{1}(\boldsymbol{k})$ decays fast enough, i.e., before the Mott insulator shells propagating inwards reach the center. From Eq. (23), we conclude that the typical time for the peak at zero wave vector of the momentum distribution function $g_{1}(k$ $=0$ ) to decay (say, one $e$-folding) is generally scaling with $1 / \sqrt{n}$, i.e., we have

$$
t_{d}=\frac{1}{U \sqrt{n}} \quad(\nu \ll 1), \quad t_{d}=\frac{1}{U} \sqrt{\frac{\nu}{n}} \quad(\nu \gg 1) .
$$

To clarify the question above without resorting to brute force numerical computation, using simple analytical means, we consider in what follows various possible modes of the disturbance propagation of the corrections to mean field, to determine the dominant, i.e., most rapid, mode of propagation.

\section{A. Propagation of sound and shock waves}

The speed of sound in a 1D optical lattice, for large filling $n$, can be derived from the dispersion law [37]

$$
\omega_{k}^{2}=4 n U J \sin ^{2}\left(\frac{k a}{2}\right)+4 J^{2} \sin ^{4}\left(\frac{k a}{2}\right),
$$

where the lattice spacing $a=\lambda / 2$ is determined by the laser wavelength $\lambda$ [the eigenvalues of $M_{\alpha \beta}$ in this case are $\lambda_{k}$ $=2 \sin ^{2}(k a / 2)$, from which, using Eq. (12), the above dispersion law follows]. This leads for $k \rightarrow 0$ to the sound speed

$$
c_{s}=a \sqrt{n U J}=\pi \sqrt{\frac{n U J}{2 m E_{R}}} .
$$

A condition for the undisturbed observability of the decay of $g_{1}(\boldsymbol{k})$ is, then, that the time for sound perturbations to propagate to the center is much larger than the decay time $t_{d}$, i.e., $R_{T F} /\left(c_{s}\right)_{0} \gg t_{d}$, where the Thomas-Fermi size of the harmonically trapped cloud $R_{T F}=\sqrt{2 m U n_{0} / \omega^{2}}$, with $n_{0}$ the central filling and central sound speed $\left(c_{s}\right)_{0}=c_{s}\left(J=J_{0}, n=n_{0}\right)$. We then obtain that, using $J_{c} \sim U / n$, 


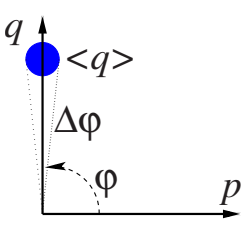

(a)

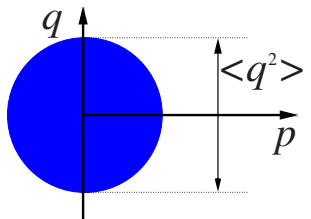

(b)
FIG. 2. (Color online) Phase space diagram (not to scale) of the coherent state (a) and the number eigenstate (b).

$$
\frac{2 U}{\pi \omega} \sqrt{\frac{E_{R}}{J}} \gg \frac{1}{\sqrt{n}} \Leftrightarrow \frac{2}{\pi \omega} \sqrt{\frac{U E_{R}}{J / J_{c}}} \gg \frac{1}{n}
$$

has to be fulfilled for a rapid quench, $\nu \ll 1$. This condition is practically always fulfilled if the optical lattice is not too tightly harmonically trapped: With a laser wavelength of $\lambda$ $=985 \mathrm{~nm}$, we have $E_{R} \simeq 3.7 \mathrm{kHz}$ for ${ }^{87} \mathrm{Rb}$ and $E_{R}$ $\simeq 8.9 \mathrm{kHz}$ for $\mathrm{Na}$, while typical values for the on-site interaction are $U \sim 10^{-2} \cdots 10^{-3} E_{R}$ [6]; furthermore, $\omega$ in the weakly confining direction(s) does not exceed $100 \mathrm{~Hz}$. For a slow quench, the right-hand side of the above inequality (37) reads $\sqrt{\nu / n}$ (respectively, $\sqrt{\nu / n}$ ) instead, and the inequality will only be fulfilled if $\nu$ is not too large, i.e., if the sweep is not too slow (as one would expect).

It is a well-established fact that shock waves in a homogeneous medium can propagate much faster than sound; we therefore need to estimate if shock waves might overtake the sound waves in the proceeding quench. However, according to the study of [39], the propagation of shock waves in the optical lattice proceeds at speeds slower than that of the sound waves for all values of $J<\frac{1}{3} n U$; the dispersion relation (35) then has negative curvature for all $k$. This condition translates into $\left(J / J_{c}\right)\left(1 / n^{2}\right) \lesssim \frac{1}{3}$. Therefore, close to the Mott transition, and for sufficiently large filling, shock waves always propagate slower than sound, as opposed to the uniform system.

\section{B. Propagation of the phase boundary between superfluid and Mott phases}

In the two examples above (sound and shock waves), we studied the propagation within a given phase (the superfluid phase). However, one might object that the motion of the superfluid-Mott phase boundary could perhaps be much faster. Clearly, the typical time scale to overcome one lattice site will again be set by the tunneling rate $J$, but this rate could possibly be enhanced (or suppressed) by a large filling factor $n \gg 1$. In general, the correct description of the propagation of a phase boundary in the Bose-Hubbard model is a very interesting and quite involved problem. In the following, we derive a rough estimate using the simpler two-site Bose-Hubbard model (a Bose-Einstein condensate Josephson junction), described by the Hamiltonian

$$
\hat{H}=J\left(\hat{a}_{1} \hat{a}_{2}^{\dagger}+\hat{a}_{1}^{\dagger} \hat{a}_{2}\right)+O(U) .
$$

In one site $\left(\hat{a}_{1}\right)$, we model the superfluid phase by a coherent state, while the other side $\left(\hat{a}_{2}\right)$ is prepared in a number eigenstate simulating the Mott phase (cf. Fig. 2). Therefore, the combined initial state factorizes

$$
\left|\psi_{12}^{0}\right\rangle=\left|\psi_{1}^{0}\right\rangle \otimes\left|\psi_{2}^{0}\right\rangle,
$$

into a coherent state $\left|\psi_{1}^{0}\right\rangle$ and a number eigenstate $\left|\psi_{2}^{0}\right\rangle$,

$$
\hat{a}_{1}\left|\psi_{1}^{0}\right\rangle=\alpha_{1}\left|\psi_{1}^{0}\right\rangle, \quad \hat{n}_{2}\left|\psi_{2}^{0}\right\rangle=n_{2}\left|\psi_{2}^{0}\right\rangle
$$

Now the question is the following: How fast does the phase coherence of the coherent state (superfluid) decay due to the coupling with the other site (Mott)? Since the propagating superfluid-Mott phase boundary is (like a shock wave) presumably very sharp, this site-to-site behavior should provide a rough estimate for the propagation speed. Of course, there will also be an on-site dephasing caused by the selfinteraction $U$, but this process precisely corresponds to the homogeneous growth of the phase fluctuations considered in the present paper and is independent of the moving phase boundary.

Without loss of generality, we assume $\alpha_{1} \in \mathbb{R}$, i.e., $\left\langle\hat{p}_{1}\right\rangle_{0}$ $=0$ and $\left\langle\hat{q}_{1}\right\rangle_{0} \neq 0$ in terms of the canonical variables $\hat{q}_{1}$ $=\left(\hat{a}_{1}^{\dagger}+\hat{a}_{1}\right) / \sqrt{2}$ and $\hat{p}_{1}=i\left(\hat{a}_{1}^{\dagger}-\hat{a}_{1}\right) / \sqrt{2}$. The initial phase uncertainty is given by (cf. Fig. 2)

$$
\left\langle\left(\Delta \hat{\varphi}_{1}\right)^{2}\right\rangle_{0}=\frac{\left\langle\hat{p}_{1}^{2}\right\rangle_{0}}{\left\langle\hat{q}_{1}\right\rangle_{0}^{2}}=O\left(\frac{1}{\alpha_{1}^{2}}\right)=O\left(\frac{1}{\left\langle\hat{n}_{1}\right\rangle}\right),
$$

which is the usual result for a coherent state. Now, if we insert the time evolution in the Heisenberg picture

$$
\hat{p}_{1}(t)=\hat{p}_{1}(0)+J t \hat{q}_{2}(0)+O\left(J^{2} t^{2}\right)
$$

we find a gradual increase of the phase variance

$$
\left\langle\left(\Delta \hat{\varphi}_{1}\right)^{2}\right\rangle(t)=\left\langle\left(\Delta \hat{\varphi}_{1}\right)^{2}\right\rangle_{0}+J^{2} t^{2} \frac{\left\langle\hat{q}_{2}^{2}\right\rangle_{0}}{\left\langle\hat{q}_{1}\right\rangle_{0}^{2}},
$$

which just corresponds to the dephasing induced by the coupling to the other site. Since the phase fluctuations of the other site are maximally large $\left\langle\hat{q}_{2}^{2}\right\rangle_{0}=O\left(n_{2}\right)$, we get together with $\left\langle\hat{q}_{1}\right\rangle_{0}=O\left(\sqrt{n_{1}}\right)$ the final result

$$
\left\langle\left(\Delta \hat{\varphi}_{1}\right)^{2}\right\rangle(t)=O\left(\frac{1}{n_{1}}\right)+O\left(J^{2} t^{2} \frac{n_{2}}{n_{1}}\right) .
$$

Hence, for equal (mean) fillings $n_{1}=n_{2}=n$, the typical dephasing time scale $\left\langle\left(\Delta \hat{\varphi}_{1}\right)^{2}\right\rangle=O(1)$ is just determined by the tunneling rate $J$ without suppression or enhancement by additional factors of $n \gg 1$.

To summarize, the propagation velocity of the two-site model for the phase boundary is of order $v_{2}=O(a J)$. The ratio of the maximal sound speed from Eq. (36) to $v_{2}$ is given by

$$
\frac{c_{s}}{v_{2}} \sim \sqrt{\frac{n U}{J}} \sim \frac{n}{\sqrt{J / J_{c}}} .
$$

It follows that sound waves are the dominant, i.e., most rapid mode of propagation of the disturbances caused by the Mott insulator shells, due to the $n$-dependent collective enhancement factor, which is absent at least from the simple two-site model for the phase boundary propagation. 


\section{EFFECTIVE SPACETIME AT LARGE WAVELENGTHS}

The freezing of the number fluctuations obtained in Sec. III A can nicely be explained via the analogy to the kinematics of quantum fields in gravity, valid at large length scales and low energies. At large wavelengths $\lambda \gg a$, the lattice structure is not important for the propagation of disturbances and the system behaves like an ordinary (possibly inhomogeneous and moving) superfluid. [This is precisely the limit in which the scaling equation (32) holds.] In the continuum limit, the excitations (phonons) possess a linear spectrum at low energies and behave in complete analogy to scalar (quantum) fields in curved spacetimes [40,41].

The case of a decreasing tunneling rate $J(t)$ is analogous to an expanding universe. This can be seen by means of the evolution equation (13) in the limit of small wave numbers $k=\sqrt{8 \lambda_{\kappa}} / a$,

$$
\left(\frac{\partial}{\partial t} \frac{1}{J(t)} \frac{\partial}{\partial t}-U n a^{2} \nabla^{2}\right) \delta \hat{n}(x, t)=0,
$$

which is identical to the wave equation for a scalar field mode in an expanding universe $[42,43]$. If the speed of sound $c_{s}^{2}(t)=U n a^{2} J(t)$ decreases fast enough (e.g., exponentially), phonons cannot propagate arbitrarily far but may only travel a finite distance. In analogy to cosmology, this corresponds to the emergence of a horizon

$$
\Delta_{h}(t)=\int_{t}^{\infty} d t^{\prime} c_{s}\left(t^{\prime}\right)=a \int_{t}^{\infty} d t^{\prime} \sqrt{J\left(t^{\prime}\right) U n} .
$$

The convergence of the above integral for $t^{\prime} \rightarrow \infty$ then indicates the emergence of an horizon. This observation matches the conclusions of Sec. III A, where we found that the solutions for $J \propto t^{-\alpha}$ with $\alpha<2$ do not freeze at late times but oscillate forever (no horizon). For an exponential sweep, on the other hand, the integral converges and a horizon exists. Because its size shrinks exponentially $\Delta_{h}(t)$ $=2 \sqrt{J_{0} U n} \exp [-\gamma t / 2] / \gamma$, it engulfs any given mode with wavelength $\lambda$ after some time. Therefore, the evolution of the phonon modes passes through three stages: oscillation $\lambda \ll \Delta_{h}\left(t_{<}\right)$, horizon crossing $\lambda=\Delta_{h}\left(t_{=}\right)$, and freezing $\lambda$ $\gg \Delta_{h}\left(t_{>}\right)$. Since each mode can be mapped to a harmonic oscillator, this evolution corresponds to the transition from underdamped to overdamped regime, which can be seen by rewriting Eq. (46) as

$$
\left(\frac{\partial^{2}}{\partial t^{2}}-\frac{j}{J} \frac{\partial}{\partial t}-J(t) U n a^{2} \nabla^{2}\right) \delta \hat{n}(x, t)=0 .
$$

Hence, identifying $J / J$ with the Hubble constant (which is indeed a constant for the exponential sweep), the freezing of the number fluctuations and the creation of a number squeezed state by the exponential sweep considered in this paper is completely analogous to cosmic inflation [42]. During this very early epoch of the cosmic evolution, the rapid expansion of space induced a squeezing of the quantum fluctuations of the inflaton scalar field - traces of these frozen and amplified quantum fluctuations can still be observed today in the anisotropies of the cosmic microwave background radiation.

\section{CONCLUSIONS}

For the Bose-Hubbard model with generally timedependent coefficients $J(t)$ and $U(t)$, we developed a rigorously controlled and number-conserving mean-field expansion for large filling $n \gg 1$ as a generalization of the original theory of Bogoliubov to the lattice. This allows us to study nonequlibrium quantum phenomena occurring in the sweep from the superfluid to the Mott phase. For two experimentally relevant cases-an exponential decay of the tunneling rate $J(t)$ and a linear increase of the interaction coupling $U(t)$, respectively - we found exact scaling solutions which facilitate fully analytical expressions for the time dependence of the Bogoliubov excitations and the resulting quantum depletion. Moreover, we were able to establish a duality between various power-law behaviors of $U(t)$ and $J(t)$, which enables transferring one solution obtained for $J=J(t), U$ $=$ const into one for $U=U(t), J=$ const, and vice versa.

In the first case (exponentially decaying tunneling rate), we observe freezing and squeezing of the initial quantum number fluctuations in complete analogy to cosmology. The final state and its energetic position between the (initial) coherent state and the Mott phase (final ground state) depends on a single adiabaticity parameter $\nu$, which is given by the ratio of the (external) sweep rate and the (internal) chemical potential. This parameter also governs the decay of offdiagonal long-range order.

Since the Bose-Hubbard model is considered a prototypical example for quantum criticality, we expect our findings to contribute to the general understanding of dynamical quantum phase transitions. Furthermore, by estimating the applicability of our results derived for the homogeneous case to the real experimental situation with harmonic trapping present, we found that the predicted effects should be observable with current optical lattice technology.

\section{ACKNOWLEDGMENTS}

This work was supported by the Emmy Noether Programme of the German Research Foundation (DFG) under Grant No. SCHU 1557/1-2,3 and by the Australian Research Council.
[1] S. Sachdev, Quantum Phase Transitions (Cambridge University Press, Cambridge, England, 2000).

[2] M. P. A. Fisher, P. B. Weichman, G. Grinstein, and D. S. Fisher, Phys. Rev. B 40, 546 (1989).
[3] D. Jaksch, C. Bruder, J. I. Cirac, C. W. Gardiner, and P. Zoller, Phys. Rev. Lett. 81, 3108 (1998).

[4] M. Greiner, O. Mandel, T. Esslinger, T. W. Hänsch, and I. Bloch, Nature (London) 415, 39 (2002). 
[5] M. Lewenstein, A. Sanpera, V. Ahufinger, B. Damski, and A. Sen (De), and U. Sen, Adv. Phys. 56, 243 (2007).

[6] O. Morsch and M. Oberthaler, Rev. Mod. Phys. 78, 179 (2006).

[7] A. Polkovnikov, S. Sachdev, and S. M. Girvin, Phys. Rev. A 66, 053607 (2002).

[8] S. R. Clark and D. Jaksch, Phys. Rev. A 70, 043612 (2004).

[9] K. Sengupta, S. Powell, and S. Sachdev, Phys. Rev. A 69, 053616 (2004).

[10] L. Isella and J. Ruostekoski, Phys. Rev. A 72, 011601(R) (2005).

[11] F. M. Cucchietti, B. Damski, J. Dziarmaga, and W. H. Zurek, Phys. Rev. A 75, 023603 (2007).

[12] J. Dziarmaga, Phys. Rev. Lett. 95, 245701 (2005).

[13] R. Schützhold, M. Uhlmann, Y. Xu, and U. R. Fischer, Phys. Rev. Lett. 97, 200601 (2006).

[14] A recent numerical study of the quench from the superfluid to the Mott side valid at low filling can be found in C. Kollath, A. M. Läuchli, and E. Altman, Phys. Rev. Lett. 98, 180601 (2007).

[15] C. Orzel, A. K. Tuchman, M. L. Fenselau, M. Yasuda, and M. A. Kasevich, Science 291, 2386 (2001).

[16] A. K. Tuchman, C. Orzel, A. Polkovnikov, and M. A. Kasevich, Phys. Rev. A 74, 051601(R) (2006).

[17] W. Li, A. K. Tuchman, H.-C. Chien, and M. A. Kasevich, Phys. Rev. Lett. 98, 040402 (2007)

[18] J. García-Ripoll, J. I. Cirac, P. Zoller, C. Kollath, U. Schollwöck, and J. von Delft, Opt. Express 12, 42 (2004).

[19] H. Fröhlich, Physica (Amsterdam) 34, 47 (1967).

[20] Y. Castin, J. Phys. IV 116, 89 (2004).

[21] M. Girardeau and R. Arnowitt, Phys. Rev. 113, 755 (1959); C. W. Gardiner, Phys. Rev. A 56, 1414 (1997).

[22] Y. Castin and R. Dum, Phys. Rev. A 57, 3008 (1998).

[23] Another possibility is the inclusion of the interaction with a large number of neighboring sites such that the inverse number of interaction partners provides the expansion parameter, see R. Schützhold, M. Uhlmann, and U. R. Fischer, e-print arXiv:0801.3984.

[24] I. Bloch, J. Dalibard, and W. Zwerger, e-print arXiv:0704.3011, Rev. Mod. Phys. (to be published).

[25] N. N. Bogoliubov, Selected Works, Part II: Quantum and Sta- tistical Mechanics (Gordon and Breach, New York, 1991).

[26] We remark that a gapped amplitude mode emerges from the rotor Hamiltonian (2) near the phase transition if one uses a dynamical Gutzwiller type mean-field ansatz for the ground state on the lattice [27], for which the condensate will be strongly depleted. Within Bogoliubov mean-field theory, i.e., using the weakly depleted superfluid ground state, such a mode does not occur, and the excitation spectrum [given by Eq. (12) in the time-independent case] always remains gapless.

[27] S. D. Huber, B. Theiler, E. Altman, and G. Blatter, Phys. Rev. Lett. 100, 050404 (2008).

[28] A. Imamoḡlu, M. Lewenstein, and L. You, Phys. Rev. Lett. 78, 2511 (1997).

[29] M. Greiner, O. Mandel, T. W. Hänsch, and I. Bloch, Nature (London) 419, 51 (2002).

[30] F. Gerbier, S. Fölling, A. Widera, O. Mandel, and I. Bloch, Phys. Rev. Lett. 96, 090401 (2006).

[31] Handbook of Mathematical Functions, edited by M. Abramowitz and I. Stegun (Dover, New York, 1970); A. Erdély, W. Magnus, F. Oberhettinger, and F. G. Tricomi, Higher Transcendental Functions (McGraw-Hill, New York, 1953), Vol. 1.

[32] D. Boers, C. Weiss, and M. Holthaus, Europhys. Lett. 67, 887 (2004).

[33] Yu. Kagan, E. L. Surkov, and G. V. Shlyapnikov, Phys. Rev. A 54, R1753 (1996).

[34] Y. Castin and R. Dum, Phys. Rev. Lett. 77, 5315 (1996).

[35] J. Plata, Phys. Rev. A 69, 033604 (2004).

[36] S. Fölling, A. Widera, T. Müller, F. Gerbier, and I. Bloch, Phys. Rev. Lett. 97, 060403 (2006).

[37] J. Javanainen, Phys. Rev. A 60, 4902 (1999).

[38] S. B. McKagan, D. L. Feder, and W. P. Reinhardt, Phys. Rev. A 74, 013612 (2006).

[39] C. Menotti, M. Kraemer, A. Smerzi, L. Pitaevskiı̆, and S. Stringari, Phys. Rev. A 70, 023609 (2004).

[40] W. G. Unruh, Phys. Rev. Lett. 46, 1351 (1981).

[41] M. Visser, Class. Quantum Grav. 15, 1767 (1998).

[42] U. R. Fischer and R. Schützhold, Phys. Rev. A 70, 063615 (2004).

[43] R. Schützhold, Phys. Rev. Lett. 95, 135703 (2005). 